\title{
On the generation of semigroups of nonlinear contractions
}

\author{
By Shinnosuke OHARU
}

(Received Dec. 10, 1969)

\section{Introduction}

Let $X$ be a real or complex Banach space and $S$ be a subset of $X$. Let $\{T(t) ; t \geqq 0\}$ be a one-parameter family of (possibly nonlinear) contractions from $S$ into itself satisfying the following conditions:

(i) $T(0)=I$ (the identity mapping), $T(t) T(s)=T(t+s)$ on $S$ for $t, s \geqq 0$;

(ii) for each $x \in S, T(t) x$ is strongly continuous in $t \geqq 0$. Then the family $\{T(t)\}$ is called a semigroup (of contractions) on $S$. And we define the infinitesimal generator $A_{0}$ of a semigroup $\{T(t)\}$ by $A_{0} x=\lim _{h \rightarrow+0} h^{-1}\{T(h) x-x\}$ and the weak infinitesimal generator $A^{\prime}$ by $A^{\prime} x=w-\lim _{h \rightarrow+0} h^{-1}\{T(h) x-x\}$, if the right sides exist, the notation "lim" (or " $w$-lim") means the strong limit (or the weak limit) in $X$.

The purpose of the present paper is to construct the semigroup of contractions determined by a (nonlinear) operator given in a Banach space. Our results consist of sufficient conditions for a (multi-valued) operator in $X$ or a pseudo-resolvent of contractions in $X$ to determine a semigroup of contractions. Also, we are concerned with the generation of semigroups of differentiable operators.

We find other interesting results on the generation of semigroups of contractions in [2]-[5], [8]-[14], in which (multi-valued) maximal dissipative, $m$-accretive or $m$-dissipative operators are treated as the infinitesimal generators. In this paper we extend these generation theorems to the case of a (multi-valued) dissipative operator $A$ such that the range $R(I-\lambda A)$ of $I-\lambda A$ contains $D(A)$ for every $\lambda>0$. Recently, Brezis and Pazy [1] considered similar problems in Hilbert spaces. A result related to their generation theorem will be given in $\S 6$.

Section 0 gives the notion of a dissipative operator and some of its basic properties.

Section 1 contains the statements of main results and some remarks.

Section 2 concerns the abstract Cauchy problem. 
Section 3 deals with the pseudo-resolvent.

Section 4 deals with the approximation of operators.

Section 5 contains the construction of the semigroup determined by the dissipative operator.

In Section 6, the differentiability of the constructed semigroup is discussed.

Finally, Section 7 deals with the construction of semigroups of differentiable operators.

The author wants to express his deep gratitude to Professors I. Miyadera and $H$. Sunouchi for their many valuable suggestions.

\section{§0. Preliminaries}

In this section we introduce some notions and notations which will be used in this paper.

An operator means a single-valued operator or a multi-valued operator when we do not specify it. For the notion of multi-valued operator, we refer to Kato $[11 ; \S 2]$ : let $A$ be an operator in $X$; then the domain $D(A)$ of $A$ is the set of all $x \in X$ such that $A x \neq \emptyset$; the range $R(A)$ of $A$ is given by $\bigcup_{x \in X} A x$; here, $A x=\emptyset$ if $x \notin D(A)$; and we write $A S$ (or $A(S)$ ) for $\bigcup_{x \in S} A x$. For $S_{1}, S_{2} \subset X, S_{1}+S_{2}$ denotes the set $\left\{x+y ; x \in S_{1}, y \in S_{2}\right\}$, where $S_{1}+S_{2}=\emptyset$ if $S_{1}=\emptyset$ or $S_{2}=\emptyset$. For a scalar $\lambda$ and $S \subset X, \lambda S$ denotes $\{\lambda x ; x \in S\}$. And we write $y+S$ for $\{y\}+S$. Accordingly, we define the sum $A+B$ of two operators $A$ and $B$ on $D(A) \cap D(B)$ by $(A+B) x=A x+B x$, the scalar multiplication $\lambda A$ on $D(A)$ by $(\lambda A) x=\lambda A x$, and the product $A B$ of two operators $A$ and $B$ by $A B x=(A B) x=A(B x)$. We write $\gamma+\lambda A$ for the operator $\gamma I+\lambda A$. And we denote by $A^{-1}$ the inverse operator of an operator $A$. Let $G(A)$ denote the graph of an operator $A$. Then $G\left(A^{-1}\right)=\{(y, x) ;(x, y) \in G(A)\}$. A single-valued operator $A$ with domain and range in $X$ is regarded as a special case of a multi-valued operator in $X$. Let $A$ be a single-valued operator such that $R(A) \subset D(A)$. Then for any positive integer $k$, we may define the iterations $A^{k}$ on $D(A)$ by $A^{k} x=A\left(A^{k-1} x\right)$. Here, we define $A^{0}=I$.

Let $A, \tilde{A}$ be two operators in $X$. Then we say that $\tilde{A}$ is an extension of $A$, and $A$ is a restriction of $\tilde{A}$ (denoted $\tilde{A} \supset A, A \subset \tilde{A}$ ), if $A x \subset \tilde{A} x$ for $x \in X$. Thus $D(A) \subset D(\tilde{A})$. Let $A$ be an operator in $X$ and $S \subset X$. Then by a restriction of $A$ to $S$, denoted $\left.A\right|_{S}$, we mean an operator such that $D\left(\left.A\right|_{S}\right)=D(A) \cap S$ and $\left.A\right|_{S} x=A x$ if $x \in S$.

Let $S \subset X$. Then we denote the closure of $S$ in $X$ by $\bar{S}$. Let $A$ be an operator in $X$. Then $B$ is called the closure of $A$ if $G(B)=\bar{G}(A)$ in $X \times X$; and we write $B=\bar{A}$.

Let $X^{*}$ be the dual space of $X$. Then we denote by $\langle x, f\rangle$ the pairing 
between $x \in X$ and $f \in X^{*}$, and the duality mapping $F$ of $X$ is the (multivalued) mapping from $X$ into $X^{*}$ defined by

$$
F(x)=\left\{f \in X^{*} ; \operatorname{re}\langle x, f\rangle=\|x\|^{2}=\|f\|^{2}\right\} .
$$

Definition 1. A dissipative operator in $X$ is an operator $A$ such that for every $x, y \in D(A)$ and $x^{\prime} \in A x, y^{\prime} \in A y$, there exists an $f \in F(x-y)$ such that

$$
\text { re }\left\langle x^{\prime}-y^{\prime}, f\right\rangle \leqq 0 \text {. }
$$

Let $S \subset X$. If an operator $A$ is dissipative and if any dissipative extension of $A$ coincides on $S$ with $A$, then it is said to be maximal dissipative on $S$. And $A$ is said to be $m$-dissipative, if it is dissipative and $R\left(I-\lambda_{0} A\right)=X$ for some $\lambda_{0}>0$.

An $m$-dissipative operator $A$ is maximal dissipative on $D(A)$. It is known (see [11; Lemma 3.5]) that if $X^{*}$ is strictly convex and if $A$ is maximal dissipative on $S$, then $A x$ is convex and closed for $x \in D(A) \cap S$. And it is proved in [8] or [15] that for an $m$-dissipative operator $A, R(I-\lambda A)=X$ for $\lambda>0$.

Definition 2. Let $S \subset X$ and $A$ be a dissipative operator. We denote by $\mathcal{E}[A ; S]$ the set of all dissipative operator $B$ such that $D(B) \subset S$ and $B x \supset A x$ for $x \in S . \quad B \in \mathcal{E}[A ; S]$ is called a maximal dissipative extension of $A$ in $S$, if it is maximal dissipative on $S$.

Definition 3. An operator $A$ is said to be demi-closed, if the following condition is satisfied: if $x_{n} \in D(A), x_{n} \rightarrow x \in X$ strongly and if there are $y_{n} \in A x_{n}$ such that $y_{n} \rightarrow y \in X$ weakly, then $x \in D(A)$ and $y \in A x$. And $A$ is said to be closed, if the graph $G(A)$ is closed in $X \times X$.

A demi-closed operator is closed. Closed linear operators, $m$-dissipative operators in a Banach space with the uniformly convex dual, and the operators treated in [16] are all demi-closed. In general, it is proved (see [11; Lemma 3.7]) that if $X^{*}$ is uniformly convex and if $A$ is maximal dissipative on $\overline{D(A)}$, then $A$ is demi-closed. This notion plays a central role in the argument of differentiability of semigroups.

Definition 4. Let $A$ be an operator in $X$. Then we define a (multivalued) operator $A^{0}$ by

$$
A^{0} x=\{y \in A x ;\|y\|=\inf [\|u\| ; u \in A x]\} .
$$

We call this operator the canonical restriction of $A$.

Let $X^{*}$ be uniformly convex and $A$ be maximal dissipative on $S$. Then $A^{0}$ is defined on $D(A) \cap S$ and $A^{0} x$ is convex and closed for each $x \in D(A) \cap S$. Assume that both $X$ and $X^{*}$ are uniformly convex and that $A x$ is convex and closed for $x \in D(A)$. Then the infimum of $\{\|y\| ; y \in A x\}$ is always attained 
by a unique element. Thus $A^{0}$ is single-valued and $D\left(A^{0}\right)=D(A)$. For details on the canonical restriction we refer to Kato $[11 ; \S 3]$.

Finally, in order to get shorter statements, we introduce the following notations.

(1) For any non-empty set $S \subset X$, we write $\|S\|$ for the infimum of $\{\|x\| ; x \in S\}$. Thus for any operator $A$ in $X,\|A x\|$ is defined for all $x \in D(A)$.

(2) Let $G$ be a single-valued operator in $X$ and $B \subset D(G)$. Then we mean by $\|G\|_{\operatorname{Lip}(B)}$ the smallest Lipschitz constant for $G$ on $B$. And we denote the family of all contractions on a fixed set $S \subset X$ by Cont $(S)$.

(3) We write $J_{\lambda}$ for the resolvent $(I-\lambda A)^{-1}$ if it is well defined. Also, we write $R_{\dot{\lambda}}$ for the range $R(I-\lambda A)=\{x-\lambda y ; y \in A x, x \in D(A)\}$ of $I-\lambda A$.

(4) Let $K \subset X$. Then $c o K$ denotes the convex hull of $K$ and $\overline{c o} K$ for the convex closure of $K$. Let $A$ be a multi-valued operator in $X$. Then we write $A_{c}$ for the operator which is defined on $D(A)$ by $A_{c} x=\overline{c o}(A x)$. For instance, if $A$ is maximal dissipative on $D(A)$, then $A=A_{c}$.

\section{$\S 1$. Main Results}

In this section we state our main results and make some remarks. The detailed statements and their proofs will be given later (see $\S \S 5,6$ ).

Throughout this paper we make the basic assumption that Banach space $X$ has the uniformly convex dual. This assumption implies each of the following ([10; Lemma 1.2]):

(a) the duality mapping $F$ of $X$ is single-valued and uniformly continuous on every bounded set of $X$;

(b) $X$ is reflexive.

The following result is well known ([10; Lemma 1.1$])$.

Proposition 1.1. An operator $A$ is dissipative if and only if $J_{\lambda}=(I-\lambda A)^{-1}$ is defined as a single-valued operator on $R_{\lambda}=R(I-\lambda A)$ and $J_{\lambda} \in \operatorname{Cont}\left(R_{\lambda}\right)$, for $\lambda>0$.

In view of this we consider the dissipative operator $A$ such that

$$
R(I-\lambda A) \supset D(A) \quad \text { for every } \lambda>0 .
$$

REMARK 1.1. (a) If $A$ is a closed dissipative operator satisfying $(R)$, then we have that

$\left(R_{c l}\right) \quad R(I-\lambda A) \supset \overline{D(A)}$ for every $\lambda>0$.

(b) Brezis and Pazy [1] treat a closed dissipative operator $A$ in a Hilbert space satisfying

$\left(R_{c o}\right) \quad R(I-\lambda A) \supset \overline{c o} D(A) \quad$ for every $\lambda>0$. 
ExAmple. Let $X=L^{2}(a, b)$. Let $A$ be an operator with domain and range in $X$, defined as follows. We denote by $\mathcal{L}^{2}(a, b)$ the class of all functions $u(s)$ on $[a, b]$ such that $u(s)$ is measurable and square summable over $[a, b]$. Let $D(A)$ be the class of those $x \in X$ for which a representative function $x(s) \in \mathcal{L}^{2}(a, b)$ can be found such that $x(s)$ is monotone non-decreasing on $[a, b],\left|x(s)-x\left(s^{\prime}\right)\right| \leqq\left|s-s^{\prime}\right|$, and $x(a)=0$. This means that

$$
x(s)=\int_{a}^{s} x^{\prime}(\sigma) d \sigma, \quad x^{\prime}(s) \in \mathcal{L}^{2}(a, b), \quad \text { and } \quad 0 \leqq x^{\prime}(s) \leqq 1 \quad \text { a. e. }
$$

We then define $A x$ as an element of $X$ for which $-x(s) x^{\prime}(s)$ is a representative function. We shall demonstrate that $A$ is a demi-closed, dissipative operator satisfying $(R)$. Let $x, y \in D(A)$. Then $-2\langle A x-A y, x-y\rangle$ can be written in the following forms:

$$
\begin{gathered}
\int_{a}^{b}(x(s)+y(s))^{\prime}(x(s)-y(s))^{2} d s+\int_{a}^{b}\left(x(s)^{2}-y(s)^{2}\right)(x(s)-y(s))^{\prime} d s, \\
-\int_{a}^{b}(x(s)-y(s))(x(s)-y(s))^{\prime} d s+\int_{a}^{b}\left[\left(x(s)^{2}-y(s)^{2}\right)(x(s)-y(s))\right]^{\prime} d s .
\end{gathered}
$$

Therefore, we have

$$
\begin{aligned}
\langle A x-A y, x-y\rangle= & -2^{-1} \int_{a}^{b}\left(x^{\prime}(s)+y^{\prime}(s)\right)(x(s)-y(s))^{2} d s \\
& -\left.2^{-1}(x(s)-y(s))^{2}(x(s)+y(s))\right|_{a} ^{b} \leqq 0,
\end{aligned}
$$

which means that $A$ is dissipative. Next, we show that $A$ is demi-closed. Assume that $x_{n} \in D(A), x, y \in X, x_{n} \rightarrow x$ strongly and $A x_{n} \rightarrow y$ weakly. We can write $2^{-1} x_{n}(s)^{2}=\int_{a}^{s} x_{n}(\sigma) x_{n}^{\prime}(\sigma) d \sigma$. But $\int_{a}^{s} x_{n}(\sigma) x_{n}^{\prime}(\sigma) d \sigma \rightarrow-\int_{a}^{s} y(\sigma) d \sigma$ for each $s \in$ $[a, b]$; the convergence on $[a, b]$ is uniform with respect to $s$. Thus we see that $x_{n}(s)^{2}$ converges uniformly to the limit $z(s)$, where $2^{-1} z(s)=-\int_{a}^{s} y(\sigma) d \sigma$. On the other hand, a subsequence $\left\{n^{\prime}\right\}$ can be found such that $x_{n^{\prime}}(s) \rightarrow x(s)$ a. e. Also, by Arzera-Ascoli's theorem there is a subsequence $\left\{n^{\prime \prime}\right\}$ of $\left\{n^{\prime}\right\}$ such that $x_{n^{\prime \prime}}(s) \rightarrow u(s)$ uniformly with respect to $s$. Clearly $u(s)$ is monotone non-decreasing, $\left|u(s)-u\left(s^{\prime}\right)\right| \leqq\left|s-s^{\prime}\right|$, and $u(a)=0$. Since $u(s)=x(s)$ a.e., $u(s)$ is a representative function of $x$, and so $x \in D(A)$. Since $x_{n^{\circ}}(s)^{2} \rightarrow u(s)^{2}, u(s)^{2}$ $=z(s)$ a. e. But $u(s)^{2}$ and $z(s)$ are continuous, so $u(s)^{2} \equiv z(s)$. Consequently, $2^{-1} u(s)^{2}=-\int_{a}^{s} y(\sigma) d \sigma$ and thus $-u(s) u^{\prime}(s)=y(s)$ a. e. This means that $A x=y$. It is clear that $D(A)$ is convex. And $D(A)$ is closed, because, as mentioned above, $x_{n} \in D(A)$, and $x_{n} \rightarrow x \in X$ strongly imply that $x \in D(A)$. Finally, we show that $R_{\lambda} \supset D(A)$ for every $\lambda>0$. For this purpose, we quote Dorroh [5; Example 4.10]: Let $\lambda>0$ and $v(s)$ be monotone nondecreasing on 
$[a, b],\left|v(s)-v\left(s^{\prime}\right)\right| \leqq\left|s-s^{\prime}\right|$, and $v(a)=0$. Then by the same argument as in [5; Example 4.10] there is a unique solution $x(s)$ of the differential equation $x(s)+x(s) x^{\prime}(s)=v(s)$ such that $x(s)$ is monotone nondecreasing on $[a, b], 0 \leqq x^{\prime}(s)$ $\leqq 1$, and $x(a)=0$. This means that $x \in D(A)$ and $(I-\lambda A) x=v$. It then follows that $R_{\lambda} \supset D(A)$.

In the sequel we shall study that a dissipative operator satisfying $(R)$ determines a semigroup of contractions. Our main results are now stated as follows.

Let $A$ be a dissipative operator satisfying (R). Then we have the following assertions.

I. There is a semigroup $\{T(t)\}$ of contractions on $\overline{D(A)}$ such that $T(t) x$

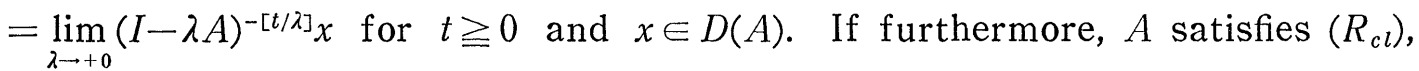
then for any $\tilde{A} \in \mathcal{E}[A ; \overline{D(A)}], T(t) x=\lim _{\lambda \rightarrow+0}(I-\lambda \tilde{A})^{-[t / \lambda]} x$ holds for $t \geqq 0$ and $x \in \overline{D(A)}$.

II. Let $\tilde{A} \in \mathcal{E}[A ; \overline{D(A)}]$ be maximal dissipative on $\overline{D(A)}$. Then there is a uniquely determined semigroup $\{T(t)\}$ of contractions on $D(\tilde{A})$ such that for each $x \in D(\tilde{A}),(d / d t) T(t) x \in \tilde{A}^{0} T(t) x$ for almost all $t \geqq 0$. If furthermore, $X$ is uniformly convex, then $A^{0}$ is the infinitesimal generator.

III. Let $A$ be single-valued and $\tilde{A} \in \mathcal{E}[A ; \overline{D(A})]$. If $\tilde{A}$ is also singlevalued and demi-closed, then it is the weak infinitesimal generator of a uniquely determined semigroup $\{T(t)\}$ of contractions on $D(\tilde{A})$.

IV. Let $X$ be uniformly convex. Let $\tilde{A} \in \mathcal{E}[A ; \overline{D(A)}]$. If $\tilde{A}$ is closed, then $\tilde{A}^{0}$ is single-valued and is the infinitesimal generator of a uniquely determined semigroup $\{T(t)\}$ of contractions on $D(\tilde{A})$. In particular, $\bar{A}^{0}$ is the infinitesimal generator of a semigroup on $D(\bar{A})$.

These theorems I-IV correspond to Theorems 5.1, 5.2, 6.1, 6.2 and 6.3.

REMARK 1.2. The results mentioned above can be extended to the case of the semigroup $\{T(t) ; t \geqq 0\}$ of Lipschitzians on a subset $S$ such that there is a real number $\omega>0$ with

$$
e^{-\omega t} T(t) \in \operatorname{Cont}(S) \quad \text { for } t \geqq 0 \text {. }
$$

This kind of semigroup is called a semigroup of local type. In this case we consider the operator $A$ satisfying the following conditions:

$$
\begin{aligned}
& A-\omega \text { is dissipative, } \\
& R(I-\lambda A) \supset D(A) \quad \text { for } \lambda \in(0,1 / \omega) .
\end{aligned}
$$

Then, by similar arguments to the analysis in the sequel we can obtain a semigroup $\{T(t)\}$ of local type on $\overline{D(A})$ and quite similar conclusions as above. 


\section{§2. Abstract Cauchy Problem}

A semigroup of contractions is closely related to the abstract Cauchy problem, formulated as follows:

$(C P)$ Given an operator $A$ in $X$ and an element $x \in X$, find a function $y(t ; x)$ such that

(i) $y(t ; x)$ is strongly absolutely continuous on every finite subinterval of $[0, \infty)$;

(ii) $y(0 ; x)=x$ and $(d / d t) y(t ; x) \in A y(t ; x)$ for a. a. $t \geqq 0$; here, if $A$ is single-valued, then " $\in$ " in the above problem is replaced by "=".

We call this an abstract Cauchy problem formulated for $A$. The multivalued operator has been introduced so that each range $R(I-\lambda A)$ may include $\overline{D(A)}$ (see also Kōmura [8]). We are led to such a kind of problem in this paper. $(C P)$ is related to the notion of semigroup in the following manner.

Proposition 2.1. Let $A$ be a dissipative operator in $X$. And suppose that for each $x \in D(A)$ there is a solution of the $(C P)$ formulated for $A$. Then there is a uniquely determined semigroup $\{T(t)\}$ of contractions on $\overline{D(A})$ in such a way that $y(t ; x)=T(t) x$ for all $t \geqq 0$ and $x \in D(A)$. In particular, if $A$ is singlevalued, then $A$ coincides with the weak infinitesimal generator of the $\{T(t)\}$ on a dense subset of $D(A)$. Conversely, if $A$ is the weak infinitesimal generator of a semigroup $\{T(t)\}$ of contractions, then $A$ is dissipative and for each $x \in D(A), T(t) x$ is a unique solution of the $(C P)$.

The proof is in Miyadera and Oharu [14]. We note that the first half of this Proposition 2.1 is still true for an arbitrary Banach space.

REMARK 2.1. Let $A$ be a dissipative operator satisfying condition $(R)$. Then, e.g., Theorem II described in $\S 1$ states that the semigroup obtained by iteration of resolvents of $A$ gives a solution operator of $(C P)$ formulated for a maximal dissipative extension $\tilde{A}$ of $A$ in $\overline{D(A)}$. In particular, if $X$ is uniformly convex and if $A$ is closed, then the semigroup gives a solution operator of $(C P)$ formulated for the canonical restriction $A^{0}$. In view of these facts, we may restate the main results in $\S 1$ in terms of abstract Cauchy problem. See also Kato [11; Remark 6.4].

ExAMPLE. Let $X=L^{2}(a, b)$ and $A$ be the operator defined in Example in $\S 1$. We are concerned with the initial-value problem

$$
u_{t}+u u_{s}=0, \quad u(0, s)=x(s) \in \mathcal{L}^{2}(a, b) .
$$

If we restrict ourselves to the initial functions $x(s)$ such that $x \in D(A)$, then as an equation in the space $X,(2.1)$ can be written

$$
(d / d t) u(t)=A u(t), \quad u(0)=x .
$$


According to Theorem III or IV stated in $\S 1$, this problem has a unique solution in $X$ if $x \in D(A)$. The solution is $u(t)=T(t) x$, where $\{T(t)\}$ is a semigroup on $D(A)$ generated by the $A$. And for each $f \in X^{*},\langle u(t), f\rangle$ is continuously differentiable and $\langle u(t), f\rangle_{t}=\langle A u(t), f\rangle$ (see Theorem 6.2 mentioned later). This means that

$$
\int_{a}^{b} u(t, \sigma) f(\sigma) d \sigma-\int_{a}^{b} x(\sigma) f(\sigma) d \sigma=-\int_{0}^{t} \int_{a}^{b} u(\xi, \sigma) u_{\sigma}(\xi, \sigma) f(\sigma) d \sigma d \xi
$$

Since it is easily seen that both $u(t, s)$ and $u_{s}(t, s)$ are measurable and essentially bounded as functions of two variables $s$ and $t$, using Fubini's theorem we obtain

$$
\int_{a}^{b}\left\{u(t, s)-x(s)+\int_{0}^{t} u(\xi, s) u_{s}(\xi, s) d \xi\right\} f(s) d s=0 .
$$

This means that for each initial function $x(s) \in \mathcal{L}^{2}(a, b)$ with $x \in D(A)$, (2.1) has a weak solution $u(t, s)$ in the sense that

$$
\begin{aligned}
& u_{t}(t, s)+u(t, s) u_{s}(t, s)=0 \quad \text { for a. a. }(t, s) ; \\
& \lim _{t \rightarrow+0} \int_{a}^{b}|u(t, s)-x(s)|^{2} d s=0 .
\end{aligned}
$$

In order to show the unicity of semigroup of contractions, we restate Proposition 2.1 in the following form.

Proposition 2.2. Suppose that $A$ is dissipative. Then there is at most one semigroup $\{T(t)\}$ of contractions on $\overline{D(A)}$ such that for $x \in D(A)$,

(a) $T(t) x \in D(A)$ for a. a. $t$; and

(b) there is a Bochner measurable, locally integrable function $f(t ; x)$ such that $f(t ; x) \in A T(t) x$ for almost all $t$ and $(d / d t) T(t) x=f(t ; x)$ almost everywhere.

\section{$\S 3 . \quad$ Pseudo-Resolvents}

In this section we consider pseudo-resolvents of nonlinear operators in $X$. Let $\left\{I_{\lambda} ; \lambda>0\right\}$ be a one-parameter family of contractions with the following properties: for every $\lambda, \mu>0$ and $x \in D\left(I_{\lambda}\right)$,

$$
\begin{aligned}
& R\left[\frac{\mu}{\lambda}+\left(1-\frac{\mu}{\lambda}\right) I_{\lambda}\right] \subset D\left(I_{\mu}\right) ; \\
& I_{\lambda} x=I_{\mu}\left[\frac{\mu}{\lambda} x+\left(1-\frac{\mu}{\lambda}\right) I_{\lambda} x\right] .
\end{aligned}
$$

We call this $\left\{I_{\lambda} ; \lambda>0\right\}$ a pseudo-resolvent of contractions. We say that a pseudo-resolvent $\left\{I_{\lambda}\right\}$ has the property $(D M)$, if there exists an $\eta>0$ such that $I_{\eta}$ has the following property: if $D\left(I_{\eta}\right) \ni x_{n} \rightarrow x$ weakly and $I_{\eta} x_{n} \rightarrow y$ 
strongly imply that $x \in D\left(I_{\eta}\right)$ and $y=I_{\eta} x$.

REMARK 3.1. $R\left(I_{\lambda}\right)$ is constant, for the resolvent equation (ii) implies that $R\left(I_{\lambda}\right) \subset R\left(I_{\mu}\right)$ for every $\lambda, \mu>0$.

Proposition 3.1. Let $A$ be a dissipative operator in $X$ and let $J_{\lambda}=(I-\lambda A)^{-1}$ for $\lambda>0$. Then we have:

(a) $\left\{J_{\lambda} ; \lambda>0\right\}$ defines a pseudo-resolvent of contractions;

(b) if $A$ is closed, then each $R_{\lambda}$ is closed; if $A$ is demi-closed, then $\left\{J_{\lambda}\right\}$ has the property $(D M)$;

(c) if $A$ satisfies $(R)$, then for $x \in D(A), J_{\lambda} x$ is strongly continuous in $\lambda>0$ and $\lim _{\lambda \rightarrow+0} J_{\lambda} x=x$.

PRoof. (a) Take any $x \in R_{\lambda}$. Then we have that

$$
R_{\mu} \supset(I-\mu A) J_{\lambda} x=J_{\lambda} x-\frac{\mu}{\lambda} \lambda A J_{\lambda} x \ni \frac{\mu}{\lambda} x+\left(1-\frac{\mu}{\lambda}\right) J_{\lambda} x,
$$

from which it follows that $R\left[\frac{\mu}{\lambda}+\left(1-\frac{\mu}{\lambda}\right) J_{\lambda}\right] \subset R_{\mu}$ and

$$
J_{\lambda} x=J_{\mu}\left[\frac{\mu}{\lambda}+\left(1-\frac{\mu}{\lambda}\right) J_{\lambda}\right] x \text {. }
$$

(b) Fix any $\lambda>0$. Suppose that $A$ is closed, $y_{n} \in R_{\lambda}$, and $y_{n} \rightarrow y \in X$ strongly. Then $x_{n}=J_{\lambda} y_{n} \rightarrow x \in X$, and so, $A x_{n} \ni\left(x_{n}-y_{n}\right) / \lambda \rightarrow(x-y) / \lambda$. Thus $(x-y) / \lambda \in A x$, which means that $y \in R_{\lambda}$. Next, assume that $A$ is demi-closed, $y_{n} \in R_{\lambda}, y_{n} \rightarrow y$ weakly, and $x_{n}=J_{\lambda} y_{n} \rightarrow x$ strongly. Then $A x_{n} \ni\left(x_{n}-y_{n}\right) / \lambda \rightarrow$ $(x-y) / \lambda$ weakly. Thus $x \in D(A)$ and $(x-y) / \lambda \in A x$, that is, $J_{\lambda} y=x$.

(c) Take any $x \in D(A)$. Since $J_{\mu} \in \operatorname{Cont}\left(R_{\mu}\right)$ for $\mu>0$, and since $\left\{J_{\lambda} x\right.$; $0<\lambda<\varepsilon\}$ is bounded, $J_{\lambda} x \rightarrow J_{\mu} x$ as $\lambda \rightarrow \mu$ by (3.1). The last part is clear from $\left\|J_{\lambda} x-x\right\| \leqq \lambda\|A x\|$.

Q.E. D.

We obtain the converse of the above Proposition 3.1.

PROPOSITION 3.2. Let $\left\{I_{\lambda} ; \lambda>0\right\}$ be a pseudo-resolvent of contractions. Then there is a dissipative operator $A$, defined on $D \equiv R\left(I_{\lambda}\right)$, such that $I_{\lambda}=$ $(I-\lambda A)^{-1}$ for every $\lambda>0$. If a $D\left(I_{\lambda}\right)$ is closed, then $A$ is closed. And if the $\left\{I_{\lambda} ; \lambda>0\right\}$ has the property $(D M)$, then $A$ is demi-closed.

PROOF. Since $R\left(I_{\lambda}\right)$ is constant by Remark 3.1, we write $D$ for it. We first show that for each $\lambda, \mu>0$ the following relation holds:

$$
\lambda^{-1}\left(x-I_{\lambda}^{-1} x\right)=\mu^{-1}\left(x-I_{\mu}^{-1} x\right) \quad \text { for } x \in D .
$$

For this purpose we show that

$$
\lambda^{-1}\left(x-I_{\lambda}^{-1} x\right) \subset \mu^{-1}\left(x-I_{\mu}^{-1} x\right)
$$

for each $x \in D$ and $\lambda, \mu>0$. Fix any $\lambda>0$ and $\mu>0$. Then, operating $I_{\mu}^{-1}$ on both sides of the resolvent equation (ii), we have that $I_{\mu}^{-1} I_{\lambda} y \ni(\mu / \lambda) y+$ 
$(1-\mu / \lambda) I_{\lambda} y$ for $y \in R_{\lambda}$, that is, $\lambda^{-1}\left(I_{\lambda} y-y\right) \in \mu^{-1}\left(I-I_{\mu}^{-1}\right) I_{\lambda} y$. Hence, for any $x \in D$ and $y \in D\left(I_{\lambda}\right)$ with $I_{\lambda} y=x$, we have $\lambda^{-1}(x-y) \in \mu^{-1}\left(I-I_{\mu}^{-1}\right) x$, which implies (3.3), We then define an operator $A$ on $D$ by $A x=\lambda^{-1}\left(x-I_{\lambda}^{-1} x\right)$ for $x \in D$. It is easy to see that $I_{\lambda}=(I-\lambda A)^{-1}$. Since $(I-\lambda A)^{-1} \in \operatorname{Cont}\left(D\left(I_{\lambda}\right)\right)$ for $\lambda>0$, Proposition 1.1 yields that $A$ is dissipative. The remaining part of the assertions is easily seen.

Q.E. D.

REMARK 3.2. Assume that each $I_{\lambda}$ of a pseudo-resolvent $\left\{I_{\lambda} ; \lambda>0\right\}$ belongs to Cont $(X)$. Then the operator $A$ obtained by Proposition 3.2 is, by definition, an $m$-dissipative operator. So, $A$ is demi-closed. Hence, $\left\{I_{\lambda}\right\}$ has the property $(D M)$. Also, $A=A_{c}$ in this case.

Next, we present an extension of Proposition 3.2 (see also Brezis and Pazy [1; Theorem 2.3]).

PROPOSITION 3.3. Let $\left\{I_{\lambda} ; \lambda>0\right\}$ be a one-parameter family of single-valued operators such that the relations (i) and (ii) hold for each $\lambda, \mu$ with $0<\mu \leqq \lambda$ and the following condition is satisfied:

(iii) for each $\lambda>0$ and $x, y \in D\left(I_{\lambda}\right)$,

$$
\left\|I_{\lambda} x-I_{\lambda} y\right\|^{2} \leqq \mathrm{re}\left\langle x-y, F\left(I_{\lambda} x-I_{\lambda} y\right)\right\rangle .
$$

Then there is a dissipative operator $A$ such that $D(A)=\bigcup_{\lambda>0} R\left(I_{\lambda}\right)$ and $(I-\lambda A)^{-1} x$ $=I_{\lambda} x$ for $x \in D\left(I_{\lambda}\right)$ and $\lambda>0$.

Proof. For each $\lambda>0$ we define an operator $A(\lambda)$ by $A(\lambda) x=\lambda^{-1}\left(x-I_{\lambda}^{-1} x\right)$ for $x \in R\left(I_{\lambda}\right)$. Then by the same argument as in the proof of (3.3), we see that

$$
R\left(I_{\lambda}\right) \subset R\left(I_{\mu}\right) \text { and } A(\lambda) \subset A(\mu) \quad \text { for } 0<\mu \leqq \lambda .
$$

Let $A=\bigcup_{\lambda>0} A(\lambda)$. Then it follows from (3.4) and (iii) that $A$ is dissipative. Also, for $\lambda>0$, we have

$$
x-\lambda A x \supset x-\lambda A(\lambda) x=I_{\lambda}^{-1} x \quad \text { for } x \in R\left(I_{\lambda}\right),
$$

so it follows that $(I-\lambda A)^{-1} y=I_{\lambda} y$ for $y \in D\left(I_{\lambda}\right)$.

Q.E. D.

COROLLARY 3.1. Let $K$ be a convex set and let $\left\{I_{\lambda} ; \lambda>0\right\}$ be a one-parameter family of single-valued operators such that $I_{\lambda} K \subset K$ for every $\lambda>0$ and (ii) and (iii) hold for every pair $\lambda, \mu$ with $0<\mu \leqq \lambda$ and $x \in K$. Then there is a dissipative operator $A$ such that $D(A)=\bigcup_{\lambda>0} I_{\lambda} K, R(I-\lambda A) \supset K \supset \operatorname{co} D(A)$ for $\lambda>0$, and $(I-\lambda A)^{-1} x=I_{\lambda} x$ for $\lambda>0$ and $x \in K$.

Proof. Set $\hat{I}_{\lambda}=\left.I_{\lambda}\right|_{K}$ for $\lambda>0$. Then $\left\{\hat{I}_{\lambda} ; \lambda>0\right\}$ satisfies all of the assumptions of Proposition 3.3. We then let $A$ be the dissipative operator obtained by Proposition 3.3. Then (3.5) states that $R(I-\lambda A) \supset R(I-\lambda A(\lambda)) \supset K$ $\supset \cup R\left(I_{\lambda}\right)=D(A)$ for $\lambda>0$.

Q.E. D. 


\section{$\S 4$. Approximation of Operators}

Let $A$ be a dissipative operator in $X$. Then by Proposition 1.1, $J_{\lambda}=(I-\lambda A)^{-1}$ exists on $R_{\lambda}=R(I-\lambda A)$ for each $\lambda>0$. We then define a single-valued operator $A_{\eta}$ on $R_{\eta}$ for $\eta>0$, by

$$
A_{\eta} x=\eta^{-1}\left[J_{\eta} x-x\right], \quad x \in R_{\eta} .
$$

It is easy to see that $\left\|A_{\eta}\right\|_{\operatorname{Lir}(R \eta)} \leqq 2 / \eta$ and that each $A_{\eta}$ is dissipative. In this section we shall give some properties of $A_{\eta}$ and some of the basic properties of closed or demi-closed dissipative operators. First, we present the next lemma which is proved in a similar way to Kato [11; $\$ 4]$ or Crandall and Pazy [2; Lemma 2.3].

Proposition 4.1. Let $A$ be a demi-closed operator in $X,\left\{x_{n}\right\} \subset D(A)$, and $x_{n} \rightarrow x_{0}$ strongly as $n \rightarrow \infty$. Then we have:

(a) Let $y_{n} \in A x_{n}$ for each $n$ and $\left\{y_{n}\right\}$ be bounded. Let $V$ be the set of all weak cluster points of $\left\{y_{n}\right\}$. Then $x_{0} \in D(A), V \neq \emptyset$ and $V \subset A x_{0}$. In particular, if $A$ is single-valued, then $A x_{0}=w-\lim _{n \rightarrow \infty} y_{n}$.

(b) Assume that $X$ is uniformly convex and that the canonical restriction $A^{0}$ is single-valued. If $y_{n} \in A x_{n}$ for each $n, x_{0} \in D\left(A^{0}\right)$ and $\lim \sup \left\|y_{n}\right\| \leqq\left\|A^{0} x_{0}\right\|$, then $A^{0} x_{0}=\lim y_{n}$.

Next, referring to Brezis and Pazy $[1 ; \S 2]$, we obtain

Proposition 4.2. Let $X$ be uniformly convex and $A$ be a closed dissipative operator satisfying $(R)$. Let $\tilde{A}$ be a maximal dissipative extension of $A$ in $\overline{D(A})$. Then $D(\tilde{A})=D(A)=D\left(A^{0}\right)$ and $\tilde{A}^{0}=A_{c}^{0}=A^{0}$.

Proof. We first note that $\tilde{A}$ is demi-closed, $\tilde{A}^{0}$ is single-valued and that $J_{\lambda} x=(I-\lambda \tilde{A})^{-1} x \rightarrow x$ strongly as $\lambda \rightarrow+0$ for $x \in D(\tilde{A})$. And $A_{c}^{0}$ is also singlevalued. Let $\eta_{k} \downarrow 0, \tilde{J}_{k} \equiv \tilde{J}_{\eta_{k}}$, and $\tilde{A}_{k} \equiv \eta_{k}^{-1}\left(\tilde{J}_{k}-I\right)$. Then by Proposition 4.1 (b),

$$
\tilde{A}^{0} x=\lim _{k \rightarrow \infty} \tilde{A}_{k} x \quad \text { for } x \in D(\tilde{A}) .
$$

Now take any $z \in D(\tilde{A})$. Then by the assumption there are $x_{k} \in D(A)$ and $y_{k} \in A x_{k}$ such that $z=x_{k}-\eta_{k} y_{k}$. Since $y_{k}=\tilde{A}_{k} z \in \tilde{A} x_{k}$, it follows that $x_{k}=\tilde{J}_{k} z$ $\rightarrow z$ strongly. Combining with (4.2), the closedness of $A$ implies that $z \in D(A)$ and $\tilde{A}^{0} z \in A z$. But then $A z \subset A_{c} z \subset \tilde{A} z$, so we see that $\tilde{A}^{0} z=A_{c}^{0} z \in A^{0} z$. Also, $\left\|A^{0} z\right\| \leqq\left\|\tilde{A}^{0} z\right\|$. Hence the relation $v \in A^{0} z \subset \tilde{A} z$ states that $v=\tilde{A}^{0} z$, because $\tilde{A}^{0}$ is single-valued. This means that $A^{0}$ is also single-valued. Q. E. D.

That $A^{0}$ is single-valued was suggested by $\mathrm{Mr}$. J. Chambers.

Let $\eta_{k} \downarrow 0, J_{k} \equiv J_{\eta_{k}}$, and $A_{k} \equiv A_{\eta_{k}}$. Then the sequence $\left\{A_{k}\right\}$ approximates $A$ in the following sense.

COROLlARY 4.1. Let $A$ be a dissipative operator satisfying $(R)$. For each $x \in D(A)$ let $V(x)$ be the set of all weak cluster points of $\left\{A_{k} x\right\}$. Then we have: 
(a) If $A$ is demi-closed, then $V(x) \neq \emptyset$ and $V(x) \subset A^{0} x$. If $A$ is demi-closed and if $A^{0}$ is single-valued, then $A^{0} x=w$ - $\lim A_{k} x$.

(b) Let $X$ be uniformly convex. If $A$ is closed, then $A^{0} x=\lim A_{k} x$ for $x \in D(A)$.

Proof. (a) For $x \in D(A), \lim J_{k} x=x$ and $\left\|A_{k} x\right\| \leqq\|A x\|$. It then follows from Proposition 4.1 (a) that $V(x) \subset A x$. Let $A_{k^{\prime}} x \rightarrow y$ weakly. Then we see that $\|y\| \leqq\|A x\|$, which means that $V(x) \subset A^{0} x$. If $A^{0}$ is single-valued, then each $V(x)$ is a singleton, and so the whole sequence $\left\{A_{k} x\right\}$ converges weakly to $A^{0} x$ for $x \in D(A)$. (b) is an immediate consequence of Proposition 4.2 and the convergence (4.2), since $\tilde{A}_{k} x=A_{k} x$ for each $x \in D(A)$ Q.E. D.

REMARK 4.1. As proved in Proposition 4.4, $\left(I-\lambda A_{k}\right)^{-1} x \rightarrow J_{\lambda} x$ strongly as $k \rightarrow \infty$, for $\lambda>0$ and $x \in D(A)$. This shows that $\left\{A_{k}\right\}$ approximates $A$ in a generalized sense.

REMARK 4.2. Let $A$ be a demi-closed dissipative operator satisfying $(R)$. Let $\tilde{A}$ be any maximal dissipative extension of $A$ in $\overline{D(A})$, and $\tilde{J}_{\lambda}=(I-\lambda \tilde{A})^{-1}$ for $\lambda>0$. Then $\tilde{J}_{\lambda} \supset J_{\lambda}$ and $\tilde{A} \supset A_{c} \supset A$. Now fix an $x \in D(A)$, then $\left\|A_{\lambda} x\right\|$ $=\lambda^{-1}\left\|J_{\lambda} x-x\right\|=\lambda^{-1}\left\|\tilde{J}_{\lambda} x-\tilde{J}_{\lambda}(x-\lambda y)\right\| \leqq\|y\|$ for $y \in \tilde{A} x$ and $\lambda>0$. Hence, $\left\|A_{\lambda} x\right\|$ $\leqq\|\tilde{A} x\| \leqq\|\overline{c o} A x\| \leqq\|A x\| \|$ for $x \in D(A)$ and $\lambda>0$. Let $J_{\lambda_{i}} x \rightarrow x$ strongly and $A_{\lambda_{i}} x \rightarrow y$ weakly. Then Corollary 4.1 (a) yields that $y \in A x$, and $\|y\| \leqq$ $\lim \inf \left\|A_{\lambda i} x\right\|$. This means that $\|A x\|=\|\tilde{A} x\|=\left\|A_{c} x\right\|=\lim _{\lambda \rightarrow+0}\left\|A_{\lambda} x\right\|$. Since $A x \subset \overline{c o} A x \subset \tilde{A} x$ for $x \in D(A)$, it follows that $A^{0} x \neq \emptyset$ and $A^{0} x \subset A_{c}^{0} x \subset \tilde{A}^{0} x$ for $x \in D(A)$.

In the following, we consider the resolvent of $A_{\eta}$.

Proposition 4.3. Let $A$ be a dissipative operator, $J_{\lambda}=(I-\lambda A)^{-1}$ for $\lambda>0$ and $A_{\eta}$ be defined by (4.1). Then for every $\eta, h>0,\left(I-h A_{\eta}\right)^{-1}$ exists as an operator from $R_{\eta+h}$ onto $R_{\eta}$, and

$$
\left(I-h A_{\eta}\right)^{-1} x=\frac{h}{\eta+h} J_{\eta+h} x+\frac{\eta}{\eta+h} x, \quad \text { for } x \in R_{\eta+h} .
$$

Proof. Fix any pair $\eta, h>0$. Then for $x \in R_{\eta_{+} h}$,

$$
\frac{h}{\eta+h} J_{\eta+h} x+\frac{\eta}{\eta+h} x=J_{\eta+h} x-\frac{\eta}{\eta+h}\left[J_{\eta+h} x-x\right] \in(I-\eta A) J_{\eta+h} x \subset R_{\eta},
$$

and for $x \in R_{\eta}$,

$$
\begin{aligned}
\left(I-h A_{\eta}\right) x & =\frac{\eta+h}{\eta} x-\frac{h}{\eta} J_{\eta} x \\
& =J_{\eta} x-\frac{\eta+h}{\eta}\left[J_{\eta} x-x\right] \in(I-(\eta+h) A) J_{\eta} x \subset R_{\eta+h},
\end{aligned}
$$

where we used the relation $h^{-1}\left[J_{h} x-x\right] \in A J_{h} x$. Using these relations, we obtain 


$$
\begin{aligned}
& \left(I-h A_{\eta}\right)\left[\frac{h}{\eta+h} J_{\eta+h} x+\frac{\eta}{\eta+h} x\right] \\
& \quad=\frac{\eta+h}{\eta}\left[\frac{h}{\eta+h} J_{\eta+h} x+\frac{\eta}{\eta+h} x\right]-\frac{h}{\eta} J_{\eta}(I-\eta A) J_{\eta+h} x=x
\end{aligned}
$$

for $x \in R_{\eta+h}$; and

$$
\begin{aligned}
& {\left[\frac{h}{\eta+h} J_{\eta+h}+\frac{\eta}{\eta+h} I\right]\left(I-h A_{\eta}\right) x} \\
& \quad=\frac{h}{\eta+h} J_{\eta+h}(I-(\eta+h) A) J_{\eta} x+\frac{\eta}{\eta+h}\left[\frac{\eta+h}{\eta} x-\frac{h}{\eta} J_{\eta} x\right]=x,
\end{aligned}
$$

for $x \in R_{\eta}$. Thus $\left(I-h A_{\eta}\right)^{-1}$ exists as an operator from $R_{\eta+h}$ onto $R_{\eta}$ and hence (4.3) holds.

Q.E. D.

PROPOSITION 4.4. Let $A$ be a dissipative operator in $X$ satisfying $\left(R_{c o}\right)$ (stated in Remark 1.1). Let $U_{\eta}=\left.A_{\eta}\right|_{\overline{c o D(A)}}$ and $J_{h}(\eta)=\left(I-h U_{\eta}\right)^{-1}$ for $\eta, h>0$. Then $R\left(I-h U_{\eta}\right) \supset \overline{c o} D(A)=D\left(U_{\eta}\right)$ and $J_{h}(\eta) \in \operatorname{Cont}\left(R\left(I-h U_{\eta}\right)\right)$ for $\eta, h>0$. Furthermore, for $x \in D(A), h>0$ and $n$,

$$
\lim _{\eta \rightarrow+0} J_{h}(\eta)^{n} x=J_{h}^{n} x
$$

Proof. Under $\left(R_{c o}\right),(4.3)$ implies that $J_{h}(\eta)[\overline{c o} D(A)] \subset \overline{c o} D(A)$ for $\eta, h>0$. Hence, we see that $R\left(I-h U_{\eta}\right) \supset \overline{c o} D(A)$. Since $U_{\eta}$ is dissipative, $J_{h}(\eta)$ $\in \operatorname{Cont}\left(R\left(I-h U_{\eta}\right)\right)$. Hence, the iterations $J_{h}(\eta)^{n}, n=1,2, \cdots$, are well-defined on $R\left(I-h U_{\eta}\right)$ and

$$
\left\|U_{\eta} J_{h}(\eta)^{n} x\right\| \leqq\|A x\| \quad \text { for } x \in D(A) .
$$

Take $x \in D(A)$, then by (4.3), $J_{h}(\eta) x-J_{h} x=\frac{h}{\eta+h} J_{\eta+h} x+\frac{\eta}{\eta+h} x-J_{h} x$ for $h, \eta>0$.

Since the right side is estimated by $\frac{h}{\eta+h}\left\|J_{\eta+h} x-J_{h} x\right\|+\frac{\eta}{\eta+h}\left\|x-J_{h} x\right\|$, Proposition 3.1 (c) implies that $\lim _{\eta \rightarrow+0} J_{h}(\eta) x=J_{h} x$. Since $J_{h}(\eta) \in \operatorname{Cont}\left(R\left(I-h U_{\eta}\right)\right)$, (4.4) holds for each positive integer $n$.

Q.E. D.

\section{$\S 5$. Construction of the Semigroups}

In this section, we construct the semigroup determined by a dissipative operator $A$ which satisfies the condition $(R)$.

LEMMA 5.1. Let $A$ be a dissipative operator in $X$ satisfying $(R)$. If $x \in D(A)$ and $T>0$, then

$$
y(t ; x)=\lim _{\lambda \rightarrow+0}(I-\lambda A)^{-[t / \lambda]} x
$$

exists uniformly for $t \in[0, T]$.

Proof. Set $J_{\lambda}=(I-\lambda A)^{-1}$ and $A_{\lambda}=\lambda^{-1}\left(J_{\lambda}-I\right), \lambda>0$. Let $x \in D(A)$ and 
$T>0$. We note that

$$
\left\|A_{h} J_{h}^{m} x\right\| \leqq\|A x\| \quad \text { for } h>0 \text { and } m .
$$

Now, assume that $n \lambda \leqq h$ and $h m \leqq T$, where $\lambda, h>0$ and $m, n$ are integers. Let $k \leqq m$. Since $J_{\lambda}^{n k} x-J_{\lambda}^{n(k-1)} x=\lambda \sum_{p=0}^{n-1} A_{\lambda} J_{\lambda}^{p} J_{\lambda}^{n(k-1)} x$, we have that $\left(J_{\lambda}^{n k} x-J_{\lambda}^{n(k-1)} x\right)$ $-\left(J_{h}^{k} x-J_{h}^{k-1} x\right)=\lambda \sum_{p=0}^{n-1}\left\{A_{\lambda} J_{\lambda}^{p} J_{\lambda}^{n(k-1)} x-A_{h} J_{h}^{k-1} x\right\}+(n \lambda-h) A_{h} J_{h}^{k-1} x$. Thus we can write

$$
\begin{aligned}
\left\langle\left(J_{\lambda}^{n k} x-J_{\lambda}^{n(k-1)} x\right)-\left(J_{h}^{k} x-J_{h}^{k-1} x\right), F\left(J_{\lambda}^{n k} x-J_{h}^{k} x\right)\right\rangle \\
=\lambda \sum_{p=0}^{n-1}\left\langle A_{\lambda} J_{\lambda}^{n(k-1)+p} x-A_{h} J_{h}^{k-1} x, F\left(J_{\lambda}^{n(k-1)+p+1} x-J_{h}^{k} x\right)\right\rangle \\
\quad+\lambda \sum_{p=0}^{n-1}\left\langle A_{\lambda} J_{\lambda}^{n(k-1)+p} x-A_{h} J_{h}^{k-1} x, F\left(J_{h}^{n k} x-J_{h}^{k} x\right)-F\left(J_{\lambda}^{n(k-1)+p+1} x-J_{h}^{k} x\right)\right\rangle \\
\quad+(n \lambda-h)\left\langle A_{h} J_{h}^{k-1} x, F\left(J_{\lambda}^{n k} x-J_{h}^{k} x\right)\right\rangle \\
\equiv I_{1}+I_{2}+I_{3} .
\end{aligned}
$$

We now estimate each term. Since $A$ is dissipative, $I_{1} \leqq 0$. Also, by using (5.2) we have that

$$
\left\|I_{2}\right\| \leqq 2\|A x\| \lambda \sum_{p=0}^{n-1}\left\|F\left(J_{\lambda}^{n k} x-J_{h}^{k} x\right)-F\left(J_{\lambda}^{n(k-1)+p+1} x-J_{h}^{k} x\right)\right\| .
$$

Employing the uniform continuity of $F$ on bounded sets, we can find a function $\varepsilon(h) \equiv \varepsilon(h ; x, T)$ such that $\varepsilon(h) \rightarrow 0$ as $h \downarrow 0$, and

$$
\sup _{n \lambda \leqq h, h k \leqq T}\left\|F\left(J_{\lambda}^{n k} x-J_{h}^{k} x\right)-F\left(J_{\lambda}^{n(k-1)+p+1} x-J_{h}^{k} x\right)\right\| \leqq \varepsilon(h) .
$$

Note that $\left\|J_{\lambda}^{n(k-1)+p+1} x-J_{\lambda}^{n k} x\right\|=0(h)$ as $h \downarrow 0$. Also,

Consequently,

$$
\left\|I_{3}\right\| \leqq|n \lambda-h|\|A x\|\left\|J_{\lambda}^{n k} x-J_{h}^{k} x\right\| \text {. }
$$

$$
\begin{aligned}
\left\|J_{\lambda}^{n m} x-J_{h}^{m} x\right\|^{2} & =\sum_{k=1}^{m}\left\{\left\|J_{\lambda}^{n k} x-J_{h}^{k} x\right\|^{2}-\left\|J_{\lambda}^{n(k-1)} x-J_{h}^{k-1} x\right\|^{2}\right\} \\
& \leqq \sum_{k=1}^{m} 2 \operatorname{re}\left\langle\left(J_{\lambda}^{n k} x-J_{h}^{k} x\right)-\left(J_{\lambda}^{n(k-1)} x-J_{h}^{k-1} x\right), F\left(J_{\lambda}^{n k} x-J_{h}^{k} x\right)\right\rangle \\
& \leqq \psi(\lambda, h),
\end{aligned}
$$

where $\phi(\lambda, h) \equiv$ const $(x, T)(h+\varepsilon(h)+m|\lambda n-h|)$ and note that $\|x\|^{2}-\|y\|^{2} \leqq$ $2 \mathrm{re}\langle x-y, F(x)\rangle$. Hence, for each $t \in[0, T]$,

$$
\left\|J_{\lambda}^{n[t / h]} x-J_{h}^{[t / h]} x\right\|^{2} \leqq \psi(\lambda, h) .
$$

First, take $\lambda=\varepsilon_{\mu}=2^{-\mu}, h=\varepsilon_{\nu}=2^{-\nu}, \quad m=\left[t / \varepsilon_{\nu}\right]$ and $n=2^{\mu-\nu}$. In this case $\psi\left(\varepsilon_{\mu}, \varepsilon_{\nu}\right)=\operatorname{const}(x, T)\left(\varepsilon_{\nu}+\varepsilon\left(\varepsilon_{\nu}\right)\right) \rightarrow 0$ as $\nu \rightarrow \infty$ and $\left|\left[t / \varepsilon_{\mu}\right]-2^{\mu-\nu}\left[t / \varepsilon_{\nu}\right]\right| \leqq 2^{\mu-\nu}$. So, 
we see that (by (5.2)) $\left\|J_{\varepsilon_{\mu}}^{\left[t / \varepsilon_{\mu}\right]} x-J_{\varepsilon_{\mu}}^{2^{\mu-\nu}\left[t / \varepsilon_{\nu}\right]} x\right\|=0\left(\varepsilon_{\nu}\right)$, and hence $\left\|J_{\varepsilon_{\mu}}^{\left[t / \varepsilon_{\mu}\right]} x-J_{\varepsilon_{\nu}}^{\left[t / \varepsilon_{\nu}\right]} x\right\|$ $\leqq 0\left(\varepsilon_{\nu}\right)+\sqrt{\psi\left(\varepsilon_{\mu}, \varepsilon_{\nu}\right)}$. This means that $\left\{J_{\varepsilon_{\nu}}^{\left[t / \varepsilon_{\nu}\right]} x\right\}$ is a Cauchy sequence. We then set

$$
y(t ; x)=\lim _{\nu \rightarrow \infty} \int_{\varepsilon_{\nu}}^{\left[t / \varepsilon_{\nu}\right]} x, \quad t \in[0, T] .
$$

Finally, we show that the limit is independent of the sequence chosen. Let $0 \leqq t<T$, and $0<\lambda \leqq h<T-t$. Taking, this time $m=[t / h]+1$ and $n=$ $\left[\frac{[t / \lambda]}{[t / h]+1}\right]$, we observe that

$$
\left\{\begin{array}{l}
m h \leqq t+h, \quad n \lambda \leqq h, \quad|t-n \lambda m| \leqq 2 \lambda+T \lambda / h, \\
|[t / \lambda]-n m| \lambda \leqq 3 \lambda+T \lambda / h, \quad m|n \lambda-h| \leqq 2 h+2 \lambda+T \lambda / h .
\end{array}\right.
$$

Again take $\lambda=\varepsilon_{\nu}$. Using (5.4) and letting $\nu \rightarrow \infty$, we see that $\phi\left(\varepsilon_{\nu}, h\right)$ $\rightarrow$ const $(x, T)(3 h+\varepsilon(h))$. Therefore, (5.3) and (5.4) imply that

$$
\left\|y(t ; x)-J_{h}^{[t / h]} x\right\| \leqq \operatorname{const}(x, T) \sqrt{h+\varepsilon(h)} .
$$

Lemma 5.2. Let $A$ be a dissipative operator in $X$ satisfying $(R)$.

(a) $\left\|y\left(t ; x_{1}\right)-y\left(t ; x_{2}\right)\right\| \leqq\left\|x_{1}-x_{2}\right\| \quad$ for $t \geqq 0$ and $x_{1}, x_{2} \in D(A)$.

(b) For every $x \in D(A)$,

$$
\left\|y(t ; x)-y\left(t^{\prime} ; x\right)\right\| \leqq\left|t-t^{\prime}\right|\|\| x \| \quad \text { for } t, t^{\prime} \geqq 0 .
$$

Proof. (a) Since $J_{\lambda} \in \operatorname{Cont}\left(R_{\lambda}\right)$ for $\lambda>0$, (a) follows from (5.3).

(b) For $x \in D(A)$, (5.2) yields that $\left\|J_{h}^{[t / h]} x-J_{h}^{\left[t^{\prime} / h\right]} x\right\| \leqq\left|t-t^{\prime}+h\right|\|A x\|$ for $t$, $t^{\prime} \geqq 0$. Letting $h \rightarrow+0$, we have (b).

Consequently, we have the following main theorem.

THEOREM 5.1. If $A$ is a dissipative operator in $X$ satisfying $(R)$, then there exists a semigroup $\{T(t)\}$ on $\overline{D(A)}$ such that

$$
T(t) x=\lim _{\lambda \rightarrow+0}(I-\lambda A)^{-[t / \lambda] x} \quad \text { for } t \geqq 0 \text { and } x \in D(A)
$$

and the convergence is uniform with respect to $t$ in every finite interval.

Proof. In view of Lemma 5.1 , set $T(t) x=y(t ; x)$ for $t \geqq 0$ and $x \in D(A)$. First, by using Lemma 3.2 (a), we can obtain a unique extension of $T(t)$ to $\overline{D(A)}$ by continuity, we denote this extension by the same symbol $T(t)$. Then each $T(t)$ maps $\overline{D(A})$ into itself and $T(t) \in \operatorname{Cont}(\overline{D(A})$ ). To establish the semigroup property ; first take $x \in D(A)$ and $t, s \geqq 0$ with $t+s \leqq T$. Let $\varepsilon>0$ and take $z \in D(A)$ such that $\|z-T(s) x\|<\varepsilon$. Then, $\|T(t+s) x-T(t) T(s) x\| \leqq \| T(t+s) x$ $-J_{\lambda}^{[(t+s) / \lambda]} x\|+\| J_{\lambda}^{[(t+s) / \lambda]} x-J_{\lambda}^{[t / \lambda]+[s / \lambda]} x\|+\| J_{\lambda}^{[t / \lambda]+[s / \lambda]} x-J_{\lambda}^{[t / \lambda]} z\|+\| J_{\lambda}^{[t / \lambda]} z-T(t) z\|+\| T(t) z$ $-T(t) T(s) x\|\leqq\| T(t+s) x-J_{\lambda}^{[(t+s) / \lambda]} x\|+2 \lambda\| A x\|+\| J_{\lambda}^{[s / \lambda]} x-z\|+\| J_{\lambda}^{[t / \lambda]} z-T(t) z\|+\| z-$ 
$T(s) x \|$. Therefore, letting $\lambda \rightarrow+0$, we have $\|T(t+s) x-T(t) T(s) x\| \leqq 2 \varepsilon$. This means that $\{T(t) ; t \geqq 0\}$ has the semigroup property. (5.6) was established in Lemma 5.1.

Q.E. D.

REMARK 5.1. Let $A$ be a dissipative operator in $X$ satisfying $\left(R_{c l}\right)$. Then $\left.J_{\lambda}^{[t / \lambda]} \in \operatorname{Cont}(\overline{D(A})\right)$ for $\lambda>0$ and $t \geqq 0$. Hence, the convergence (5.6) holds for all $t \geqq 0$ and $x \in \overline{D(A)}$.

REMARK 5.2. (a) Let $A$ be a dissipative operator satisfying $(R)$, and $\{T(t)\}$ be the corresponding semigroup obtained by Theorem 5.1. Let $\tilde{A} \in \mathcal{E}[A ; \overline{D(A)}]$ and put $\tilde{R}_{\lambda}=R(I-\lambda \tilde{A})$ and $\tilde{J}_{\lambda}=(I-\lambda \tilde{A})^{-1}$, then $\tilde{J}_{\lambda} \supset J_{\lambda}$ for $\lambda>0$. Now, if $\tilde{A}$ is maximal dissipative on $\overline{D(A)}$, then by Remark 1.1 (a) we have that $\tilde{R}_{\lambda} \supset \overline{D(A)} \supset D(\tilde{A})$ for $\lambda>0$. So that $\tilde{A}$ satisfies $\left(R_{c l}\right)$. Thus, Lemma 5.1 and Remark 5.1 yield that

$$
T(t) x=\lim _{\lambda \rightarrow+0}(I-\lambda \tilde{A})^{-[t / \lambda]} x
$$

for $t \geqq 0$ and $x \in \overline{D(A)}$.

(b) Let $A$ be a dissipative operator satisfying $\left(R_{c l}\right)$. Then for $\tilde{A} \in \mathcal{E}[A$; $\overline{D(A)}], \widetilde{R}_{\lambda} \supset \overline{D(A)} \supset D(\tilde{A})$ for $\lambda>0$, and hence we have the same conclusion (5.6) for $t \geqq 0$ and $x \in \overline{D(A)}$.

In view of these results, we obtain the following

THEOREM 5.2. Let $A$ be a dissipative operator in $X$ satisfying $\left(R_{c l}\right)$. Then there is a semigroup $\{T(t)\}$ of contractions on $\overline{D(A)}$ such that the convergence (5.7) holds for $\tilde{A} \in \mathcal{E}[A ; \overline{D(A)}]$.

Next, in terms of pseudo-resolvent, we can obtain some variations of Theorem 5.1.

COROLlaRY 5.1. Let $S \subset X$, and $\left\{I_{\lambda} ; \lambda>0\right\}$ be a pseudo-resolvent of contractions from $S$ into itself. Then there is a semigroup $\{T(t)\}$ of contractions on $R\left(I_{\lambda}\right)$ such that $T(t) x=\lim _{\lambda \rightarrow+0} I_{\lambda}^{[t / \lambda]} x$ for each $t \geqq 0$ and $x \in R\left(I_{\lambda}\right)$.

The proof is a simple consequence of Theorem 5.1. For, Proposition 3.2 implies that $\left\{I_{\lambda}\right\}$ determines a dissipative operator $A$ on $D \equiv R\left(I_{\lambda}\right)$ in such a way that $I_{\lambda}=(I-\lambda A)^{-1}$. The assumptions yield that $A$ satisfies condition $(R)$ and therefore, by Theorem 5.1 we have the assertions.

COROLlARY 5.2. Let $K \subset X$ be a convex set and $\left\{I_{\lambda} ; \lambda>0\right\}$ be a one-parameter family of single-valued operators such that $I_{\lambda} K \subset K$ for every $\lambda>0$ and such that

$$
\begin{gathered}
I_{\lambda} x=I_{\mu}\left[\frac{\mu}{\lambda} x+\left(1-\frac{\mu}{\lambda}\right) I_{\lambda} x\right], \\
\left\|I_{\lambda} x-I_{\lambda} y\right\|^{2} \leqq \mathrm{re}\left\langle x-y, F\left(I_{\lambda} x-I_{\lambda} y\right)\right\rangle,
\end{gathered}
$$

for $\lambda, \mu$ with $0<\mu \leqq \lambda$ and $x \in K$. Then there exists a semigroup $\{T(t)\}$ of 
contractions on $\overline{\bigcup_{\lambda>0} I_{\lambda} K}$ such that $T(t) x=\lim _{\lambda \rightarrow+0} I_{\lambda}^{[t / \lambda] x}$ for $t \geqq 0$ and $x \in \bigcup_{\lambda>0} I_{\lambda} K$.

The proof is easily seen from Corollary 3.1 and Theorem 5.1, in the similar way to the proof of Corollary 5.1.

Finally, we present a result on the semigroups determined by the approximate operators $A_{\eta}$.

COROLlary 5.3. Let $A$ be a dissipative operator in $X$ satisfying $\left(R_{c o}\right)$, and $\{T(t)\}$ be the corresponding semigroup on $\overline{D(A)}$. Let $A_{\eta}, \eta>0$, be the operators defined by (4.1). Then we have:

(a) Let $\left.U_{\eta} \equiv A_{\eta}\right|_{\overline{c o D}(A)}$ for $\eta>0$, then

$$
T_{\eta}(t) x=\lim _{\lambda \rightarrow+0}\left(I-\lambda U_{\eta}\right)^{[t / \lambda]} x
$$

exists for $t \geqq 0, x \in \overline{c o} D(A)$ and $\eta>0$, and $\left\{T_{\eta}(t) ; t \geqq 0\right\}$ forms a semigroup of contractions on $\overline{c o} D(A)$. Furthermore, if $x \in D(A)$, then the convergence is uniform with respect to $\eta>0$ and $t$ in every finite interval.

(b) For $x \in \overline{D(A)}$ and $t \geqq 0$,

$$
\lim _{\eta \rightarrow+0} T_{\eta}(t) x=T(t) x,
$$

where the convergence is uniform with respect to $t$ in every finite interval.

Proof. (a) The first assertion follows from Proposition 4.4 and Remark 5.1. To prove the second assertion; let $x \in D(A)$ and $T>0$, then by (4.5) we see that the set $\left\{J_{h}(\eta)^{n} x ; h, \eta>0, n h \leqq T\right\}$ is bounded and $\sup _{0 \leqq p \leqq n-1} \| J_{\lambda}(\eta)^{n(k-1)+p+1} x$ $-J_{\lambda}(\eta)^{n k} x\|\leqq n \lambda\| A x \|$. Therefore, by the same argument as in Lemma 5.1, we have the following estimate:

$$
\left\|T_{\eta}(t) x-J_{h}(\eta)^{[t / h]} x\right\| \leqq \text { const }(x, T) \sqrt{h+\varepsilon(h)},
$$

where the constant const $(x, T)$ and the function $\varepsilon(h)$ are independent of $\eta>0$ and $t \in[0, T]$.

(b) Let $x \in D(A)$ and $T>0$. Then (5.5) and (5.10) imply that

$$
\left\|T(t) x-T_{\eta}(t) x\right\| \leqq \operatorname{const}(x, T) \sqrt{h+\varepsilon(h)}+\left\|J_{h}^{[t / h]} x-J_{h}(\eta)^{[t / h]} x\right\| .
$$

Take any $\varepsilon>0$, then there is an $h_{0}>0$ such that const $(x, T) \sqrt{h_{0}+\varepsilon\left(h_{0}\right)}<\varepsilon / 2$. Since $\left\{\left[t / h_{0}\right] ; 0 \leqq t \leqq T\right\}$ is a finite set, there is an $\eta_{0}$ such that $\| J_{h_{0}}^{\left[t / h_{0}\right]} x$ $-J_{h_{0}}(\eta)^{\left[t / h_{0}\right]} x \|<\varepsilon / 2$ for $\eta \in\left(0, \eta_{0}\right)$. Consequently, $\sup _{0 \leqq t \leqq T}\left\|T(t) x-T_{\eta}(t) x\right\| \leqq \varepsilon$ for $\eta \in\left(0, \eta_{0}\right)$.

Q.E. D.

\section{§ 6. Differentiability of Semigroups}

In this section we consider the differentiability of semigroups obtained by Theorem 5.1 and some relations among the semigroups and the infinitesi- 
mal generators. The central part of the proof is based on the results by Kato $[10,11]$.

Throughout this section we use the following notations: Let $r_{n}=2^{n}$, and $I_{r}=[0, r]$ for $r>0$. We then set $J_{n}=\left(I-r_{n}^{-1} A\right)^{-1}, A_{n}=r_{n}\left[J_{n}-I\right]$, and $T(n ; t)$ $=\left(I-r_{n}^{-1} A\right)^{-\left[t r_{n}\right]}$.

LEMMA 6.1. Let $A$ be a dissipative operator satisfying $(R)$. Let $x \in D(A)$ and $f_{n}(t)=A_{n} T(n ; t) x$ for $t \geqq 0$. Then,

$$
\begin{gathered}
f_{n}(t) \in A J_{n} T(n ; t) x \text { and }\left\|f_{n}(t)\right\| \leqq\|A x\| \quad \text { for } t \geqq 0, \\
\left\|[T(n ; t)-I] J_{n} x-\int_{0}^{t} f_{n}(s) d s\right\|=0\left(1 / r_{n}\right) .
\end{gathered}
$$

Proof. (6.1) is obvious. (6.2) is obtained by estimating

$$
\begin{aligned}
\int_{0}^{\left[r_{n} t\right] / r_{n}} f_{n}(s) d s & =r_{n}^{-1} \sum_{k=1}^{[r n t]} A_{n} J_{n}^{(k-1)} x \\
& =[T(n ; t)-I] J_{n} x+r_{n}^{-1}\left\{A_{n} x-A_{n} T(n ; t) x\right\} \text {. Q. E. D. }
\end{aligned}
$$

LEMMA 6.2. Let $A$ be a demi-closed dissipative operator satisfying $(R)$. Let $\{T(t)\}$ be the semigroup of contractions on $\overline{D(A)}$, obtained by Theorem 5.1. Then we have:

(a) For every $x \in D(A), T(t) x \in D(A)$ for $t \geqq 0$ and there is a function $f(\cdot ; x)$ on $[0, \infty)$ such that $f(t ; x) \in A_{c}^{0} T(t) x$ for almost all $t \geqq 0$ and

$$
T(t) x-x=\int_{0}^{t} f(s ; x) d s, \quad \text { for } t \geqq 0 .
$$

(b) If $A$ is single-valued, then for $x \in D(A), A T(t) x$ is weakly continuous in $t \geqq 0$, and

$$
T(t) x-x=\int_{0}^{t} A T(s) x d s, \quad \text { for } t \geqq 0 .
$$

Proof. (a) Fix any $x \in D(A)$ and any $p$ with $1<p<+\infty$. And set $f_{n}(t)=A_{n} T(n ; t) x$. Then by $(6.1)\left\{\left.f_{n}\right|_{I_{r}} ; n\right\}$ forms a bounded set of $L^{p}\left(I_{r} ; X\right)$ for each integer $r>0$. Thus, by moving $r$ and by using the diagonal procedure, we may find a subsequence $\{q\}$ of $\{n\}$ and a function $f$, defined on $[0, \infty)$, such that $f_{q} \mid I_{r}$ converges to $f \mid I_{r}$ weakly in $L^{p}\left(I_{r} ; X\right)$, for each $r$. Hence, $x * \int_{0}^{t} f_{q}(s) d s \rightarrow x * \int_{0}^{t} f(s) d s$ for $x^{*} \in X^{*}$ and $t \geqq 0$. Therefore (6.4) follows from (6.2). Next, we write $V(t)$ for the set of all weak cluster points of $\left\{f_{n}(t) ; n\right\}$ for each $t$. Then from Lemma 5.1 and Proposition 4.1 (a) it follows that $T(t) x \in D(A), V(t) \neq \emptyset$, and $V(t) \subset A T(t) x$ for $t \geqq 0$. Hence, by the same argument as in Kato [11; Lemma 8.2] we see that $f(t) \in \overline{c o} A T(t) x$ almost everywhere. Moreover, in a similar way to Kato [11; Lemma 6.2], we can 
prove that $\|f(t)\| \leqq\|\overline{c o} A T(t) x\|$ almost everywhere (where we apply the argument in Remark 4.2). Thus it follows that $f(t) \in A_{c}^{0} T(t) x$ almost everywhere.

(b) Aussume that $A$ is single-valued. Then each $V(t)$ is a singleton, and so, Proposition 4.1 implies that $w$ - $\lim f_{n}(t)=A T(t) x$ for all $t \geqq 0$. Also, from the strong continuity of $T(t) x$ and the boundedness of $A T(t) x$ we see that $A T(t) x$ is weakly continuous in $t$. Thus (6.5) follows from (6.2). Q. E. D.

REMARK 6.1. Let $A$ be a demi-closed dissipative operator satisfying $(R)$ and $\{T(t)\}$ the semigroup obtained by Theorem 5.1. Then $\left\{\left.T(t)\right|_{D(A)} ; t \geqq 0\right\}$ forms a semigroup of contractions on $D(A)$ by the above lemma.

REMARK 6.2. By (6.4) we see that the infinitesimal generator $A_{0}$ of $\{T(t)\}$ is densely defined in $D(A)$.

In view of these results, we obtain the following.

First, combining with Remark 5.2 (a) we obtain

Theorem 6.1. Let $A$ be a dissipative operator satisfying $(R)$. Let $\tilde{A}$ be any maximal dissipative extension of $A$ in $\overline{D(A)}$. Then there is a uniquely determined semigroup $\{T(t)\}$ of contractions on $D(\tilde{A})$ such that for each $x \in D(\tilde{A})$, $(d / d t) T(t) x \in \tilde{A}^{0} T(t) x$ for almost all $t \geqq 0$.

Next, for the single-valued case, we obtain

THEOREM 6.2. Let $A$ be a single-valued, demi-closed dissipative operator satisfying $(R)$. Then $A$ is the weak infinitesimal generator of a unique semigroup $\{T(t)\}$ of contractions on $D(A)$ such that for each $x \in D(A), T(t) x$ is weakly continuously differentiable in $t \geqq 0$ and $T(t) x-x=\int_{0}^{t} A T(s) x d s$ for $t \geqq 0$.

In the remainder of this section we consider the case $X$ is uniformly convex. Assume that $X$ is uniformly convex. Let $A$ be a closed dissipative operator satisfying $(R)$, and $\tilde{A}$ be any maximal dissipative extension of $A$ in $\overline{D(A)}$. Then by Theorem 6.1, $\tilde{A}$ generates a semigroup $\{T(t)\}$ of contractions on $D(\tilde{A})$. Also, from Proposition 4.2 it follows that $D(\tilde{A})=D(A)$ and $\tilde{A}^{0}=A^{0}$. Hence, for $x \in D(A),\|\tilde{A} T(t) x i\|=\|A T(t) x\|$ for $t \geqq 0$ and $(d / d t) T(t) x=A^{0} T(t) x$ almost everywhere. Therefore, we can say that $\{T(t)\}$ is the semigroup on $D(A)$ which is determined by the $A$. For this semigroup, we have the following

LEMMA 6.3. For each $x \in D(A)$, we have:

(a) $\|A T(t) x\|$ is of bounded variation on every finite interval of the form $[0, T]$ and has no positive jumps;

(b) the right derivative $D^{+} T(t) x$ exists and strongly right-continuous in $t$, and $D \cdot T(t) x=A^{0} T(t) x$ for all $t \geqq 0$;

(c) $A^{0} T(t) x$ is strongly continuous except possibly at a countable number of points $t$.

Proof. (a) Let $\tilde{A}$ be a maximal dissipative extension of $A$ in $\overline{D(A)}$. Let 
$x \in D(A)$ and $0 \leqq r<t$. Then by the same argument as in Kato [11; Lemma 6.6] we see that $\|A T(t) x\| \leqq\|A T(r) x\|$.

(b) By Proposition 4.2, $\tilde{A}^{0}\left(=A^{0}\right)$ is a single-valued operator with $D\left(\tilde{A}^{0}\right)$ $=D(A)$. Fix any $x \in D(A)$ and $t \geqq 0$. And choose a sequence $t_{k} \downarrow t$. Then by the proof of Kato [11; Theorem 7.5$]$ we see that $\left\{\tilde{A}^{0} T\left(t_{k}\right) x\right\}$ contains a subsequence which converges strongly to $\tilde{A}^{0} T(t) x$. So, $\tilde{A}^{0} T(t) x$ is strongly right-continuous in $t$. But, since $T(t) x-x=\int_{0}^{t} \tilde{A}^{0} T(s) x d s$ by Theorem 6.2, it follows that $D^{+} T(t) x=\tilde{A}^{0} T(t) x=A^{0} T(t) x$ for each $t$.

(c) By (a), $\left\|\tilde{A}^{0} T(t) x\right\|=\|A T(t) x\|$ is continuous except for a countable number of points $t$. In order to prove that $\tilde{A}^{0} T(t) x$ is continuous except for those points, it suffices to repeat the same argument as in (b) with $t_{k} \uparrow t$.

Q.E.D.

Hence, we obtain the following result.

THEOREM 6.3. Assume that $X$ is uniformly convex. Let $A$ be a closed dissipative operator satisfying $(R)$. Then $A^{0}$ is the infinitesimal generator of a unique semigroup $\{T(t)\}$ of contractions on $D(A)$ such that for each $x \in D(A)$, $D^{+} T(t) x=A^{0} T(t) x$ for all $t \geqq 0$ and $D^{+} T(t) x$ is strongly right-continuous in $t \geqq 0$.

REMARK 6.3. Let $X$ be a Hilbert space. Then there are the following remarkable results (see Crandall and Pazy [2, 3], and Kōmura [9]):

(I) If $A$ is maximal dissipative in $X$, then $\overline{D(A)}$ is convex.

(II) For any dissipative operator $A$ in $X$ there is a unique $m$-dissipative extension $\tilde{A}$ of $A$ such that $D(\tilde{A}) \subset \overline{c o} D(A)$.

In view of these results, we see that Theorem 6.3 gives a result of Brezis and Pazy [1; Theorem 2.1].

Finally, we present some variations of Theorem 6.3 in terms of pseudoresolvent:

COROLlary 6.1. Assume that $X$ is uniformly convex. Let $S$ be a closed subset of $X$ and $\left\{I_{\lambda} ; \lambda>0\right\}$ be a pseudo-resolvent of contractions from $S$ into itself. Then there are a closed dissipative operator $A$ defined on $D \equiv R\left(I_{\lambda}\right)$ and a semigroup $\{T(t)\}$ on $D$, such that $I_{\lambda}=(I-\lambda A)^{-1}$ for $\lambda>0$ and $A^{0}$ is the infinitesimal generator of $\{T(t)\}$.

Proof. Let $A$ be the closed dissipative operator obtained by Proposition 3.2. Then it satisfies the conditions of Theorem 6.3.

COROLlary 6.2. Let $K \subset X$ be convex, and $\left\{I_{\lambda} ; \lambda>0\right\}$ be a family of single-valued operators satisfying all of the assumptions of Corollary 5.2. Then there is a closed dissipative operator $A$ such that $\overline{D(A})=\bigcup_{\lambda>0} I_{\lambda} K, I_{\lambda} x=(I-\lambda A)^{-1} x$ for $\lambda>0$ and $x \in K$, and such that $A^{0}$ is the infinitesimal generator of a unique semigroup on $D(A)$.

Proof. Let $A$ be the closure of the dissipative operator obtained by 
Corollary 3.1. Then it satisfies the assumptions of Theorem 6.3, Q. E. D.

\section{$\S 7$. Semigroups of Differentiable Operators}

The purpose of this section is to construct the semigroups of differentiable operators.

Let $S \subset X$ and $G$ be a single-valued operator in $X$ such that $D(G) \supset S$ and there is an $L(X, X)$-valued continuous function $d G(\cdot)$ on $S$ such that

$$
\|G(x+y)-G x-d G(x) y\|=o(\|y\|)
$$

for $x \in S$ and $y \in X$ with $x+y \in S$. Here $L(X, X)$ denotes the Banach algebra of endomorphisms of $X$. Then we denote the family of such operators by $F(S)$. lf $S$ is open, then $F(S)$ is the family of operators which are continuously Fréchet $(F-)$ differentiable in $S$. For the notions of $F$-differentiable operators, Gateaux (G-) differentiable operators, and analytic operators, we refer to Hille-Phillips [7; Chap. III] and J. T. Schwartz [19]. (By an analytic operator we mean an analytic function on vectors to vectors.)

In the remainder of this section we need to assume that $X$ is a complex Banach space. Our result is the following

THEOREM 7.1. Let $A$ be a single-valued, closed dissipative operator satisfying $(R)$. Assume that $\overline{D(A)}$ is the closure of an open convex set $D$ (hence $\left(R_{c o}\right)$ is satisfied) and that $(I-\lambda A)^{-1}$ is G-differentiable in $D$ for every $\lambda>0$. Let $\{T(t)\}$ be the semigroup on $\overline{D(A)}$ obtained by Theorem 5.1. Then we have:

(a) for each $t>0, T(t)$ is analytic in $D$ and $T(t) \in F(D)$;

(b) for each $x \in D \cap D(A), A T(t) x$ is strongly continuous in $t \geqq 0$ and $(d / d t) T(t) x=A T(t) x=d T(t)(x) A x$ for all $t \geqq 0$.

Before proving this theorem, we state some remarks and give an example.

REMARK 7.1. Let $\{T(t)\}$ be a $\left(C_{0}\right)$-semigroup of bounded linear operators and $A$ be the infinitesimal generator. Then for each $x \in D(A),(d / d t) T(t) x$ $=A T(t) x=T(t) A x$ for $t \geqq 0$. The assertion (b) corresponds to this property and the nonlinearity appears in the term $d T(t)(x)$.

REMARK 7.2. If a single-valued operator $G$ on a complex Banach space $X$ is uniformly Lipschitz continuous and $G$-differentiable on $X$, then by virtue of the Liouville's theorem for vector-valued analytic functions $G$ becomes a combination of a linear operator and a translation by a constant vector. Now, assume that $A$ is a densely defined, $m$-dissipative operator in $X$ and $(I-\lambda A)^{-1}$ is $G$-differentiable in $X$ for $\lambda>0$. Then $A$ determines a semigroup $\{T(t)\}$ of contractions on $X$, and Theorem 7.1 states that each $T(t)$ is an analytic operator on $X$. Therefore, it is proved that for each $t \geqq 0, x \rightarrow S(t) x=$ 
$T(t) x-T(t) 0$ is a linear operator on $X$ (see Kömura $[9 ; \S 4$, p. 397]). Since $\|S(t) x\| \leqq\|x\| \quad$ and $\quad S(t) S(s) x=S(t) T(s) x-S(t) T(s) 0=T(t+s) x-T(t+s) 0=S(t+s) x$ for $t, s \geqq 0$ and $x \in X$, the family $\{S(t) ; t \geqq 0\}$ forms a $\left(C_{0}\right)$-semigroup of linear contractions on $X$. Therefore $\{T(t)\}$ is an affine semigroup (see Crandall and Pazy $[2 ; \S 5])$.

EXAMPle. Let $X=C^{1}$ and let us consider an operator $A$ defined by $A z=z^{2}, z \in D(A)=\left\{z \in C^{1} ; \operatorname{re} z \leqq-|\operatorname{im} z|\right\}$. Then the interior $D(A)^{0}=\left\{z \in C^{1}\right.$; re $z<-|\operatorname{im} z|\}$ is convex. Since re $\left(z_{1}^{2}-z_{2}^{2}\right)\left(\overline{z_{1}-z_{2}}\right)=\left|z_{1}-z_{2}\right|^{2}$ re $\left(z_{1}+z_{2}\right) \leqq 0$ for $z_{1}, z_{2} \in D(A), A$ is dissipative. And we see that $R_{\lambda} \supset D(A)$ for every $\lambda>0$. Since $z \rightarrow z-\lambda z^{2}$ is analytic on $D(A)^{0}$, it follows from the implicit function theorem that $(I-\lambda A)^{-1}$ is analytic in $D(A)^{0}$.

In the remainder of this section, let $A$ be a single-valued, closed dissipative operator satisfying all of the assumptions of Theorem 7.1. Let $U_{\eta}=A_{\eta} \mid \overline{D(A)}$ for $\eta>0$, and $\left\{T_{\eta}(t)\right\}$ be the semigroup on $\left.\overline{D(A}\right)$ determined by $U_{\eta}$ in the sense of Corollary 5.3. And, taking a sequence $\eta_{m} \downarrow 0$, we write $U_{m}$ for $U_{\eta_{m}}$ and $\left\{T_{m}(t)\right\}$ for $\left\{T_{\eta_{m}}(t)\right\}$.

For the proof of Theorem 7.1 we need the theory of analytic functions on vectors to vectors. Let $G$ be an operator in $X$, which is $G$-differentiable in a finitely open set $D$, and if $x \in D$ we may define the $n$-th variation $\delta^{n} G(x ; v)$ of $G$ at $x$ with increment $v$ as

$$
\delta^{n} G(x ; v)=\left[\frac{d^{n}}{d \xi^{n}} G(x+\xi v)\right]_{\xi=0} .
$$

The key fact is the following theorem (see Hille-Phillips [7; Theorem 3.18.1]).

THEOREM A. Let $\left\{G_{n}\right\}$ be a sequence of operators in $X$ analytic and locally uniformly bounded in a fixed domain $D$. If $G x=\lim G_{n} x$ exists in $D$, then the limit operator $G$ is analytic in $D$. Furthermore, $\delta^{k} G(x ; v)=\lim \delta^{k} G_{n}(x ; v)$ for each $k, x \in D$ and $v \in X$.

LEMMA 7.1. (a) For each $\lambda>0, J_{\lambda}=(I-\lambda A)^{-1}$ is analytıc in $D$ and for $x \in D \cap D(A)$ and $y \in X, \lim _{\lambda \rightarrow+0} d J_{\lambda}(x) y=y$.

(b) For each $x \in D \cap D(A), \lim _{\eta \rightarrow+0} U_{\eta} x=A x$.

ProOF. Since each $J_{\lambda}$ is locally bounded in $\mathrm{D}$, it is analytic in $D$. Since $\lim _{\lambda \rightarrow+0} J_{\lambda} x=x$ for $x \in D(A)$, Theorem A implies that $d J_{\lambda}(x) y=\delta J_{\lambda}(x ; y) \rightarrow y$ strongly as $\lambda \rightarrow+0$, for $x \in D \cap D(A)$ and $y \in X$.

(b) Fix an $x \in D \cap D(A)$. Then $x-\eta A x \in D$ for $\eta>0$ sufficiently small. Since $U_{\eta} x=\eta^{-1}\left[J_{\eta} x-J_{\eta}(x-\eta A x)\right]$, it follows from the argument in Hille-Phillips [7; Theorem 3.17.1, p. 113] that $\left\|U_{\eta} x-d J_{\eta}(x) A x\right\|=\eta^{-1} o(\|A x\| \eta)$ as $\eta \rightarrow+0$. Therefore, combining with (a) we have the assertion.

Q. E. D.

LemmA 7.2. (a) For each $t \geqq 0, T_{m}(t)$ and $T(t)$ are analytic in $D$ (hence $\in F(D))$, and $\lim d T_{m}(t)(x) y=d T(t)(x) y$ for $x \in D$ and $y \in X$. 
(b) For each $x \in D, y \in X, d T(t)(x) y$ is strongly continuous in $t$.

PROOF. (a) For the proof of (a) it suffices to show that $T_{m}(t)$ are analytic and locally uniformly bounded in $D$. For if so, then Corollary 5.3 (b) and Theorem A would yield that $T(t)$ is analytic in $D$ (hence $T(t) \in F(D)$ ) and $d T_{m}(t)(x) y=\delta T_{m}(t)(x ; y) \rightarrow \delta T(t)(x ; y)=d T(t)(x) y$ strongly as $m \rightarrow \infty$ for $x \in D$ and $y \in X$. Now, fix an $\eta>0$. Then (4.3) and Lemma 7.1 (a) imply that $\left(I-h U_{\eta}\right)^{-1}$ maps $D$ into itself and is analytic in $D$ (and hence $\in F(D)$ ). Thus $\left(I-h U_{\eta}\right)^{-n} \in F(D)$ for $h>0$ and $n$, by the chain rule of $F$-differentiability. On the other hand, (4.5) implies that for any $T>0$, the contractions $\left\{\left(I-h U_{\eta}\right)^{-n}\right.$; $h, \eta>0, n h \in[0, T]\}$ are locally uniformly bounded in $D$. Hence, by Corollary 5.3 (a) and Theorem A we see that $T_{m}(t)$ are analytic and locally uniformly bounded in $D$.

(b) Fix any $x \in D$ and $y \in X$ with $\|y\|=1$. Let $\rho(x ; y)$ be the supremum of all numbers $\rho$ such that $|\xi| \leqq \rho$ implies that $x+\xi y \in D$, and $C$ be any circle $|\xi|=\rho^{\prime}<\rho(x ; y)$. Then by the Cauchy's integral formula [19; p. 111],

$$
d T(t)(x) y=\frac{1}{2 \pi i} \int_{C} \xi^{-2} T(t)(x+\xi y) d \xi, \quad \text { for } t \geqq 0 .
$$

Now, let $0 \leqq t, t^{\prime} \leqq T$, then we have that

$$
d T\left(t^{\prime}\right)(x) y-d T(t)(x) y=\frac{1}{2 \pi i} \int_{C} \xi^{-2}\left\{T\left(t^{\prime}\right)(x+\xi y)-T(t)(x+\xi y)\right\} d \xi .
$$

Since the integrand is uniformly bounded with respect to $\xi \in C$ and $t, t^{\prime} \in$ $[0, T]$, and since the convergence $\lim _{t^{\prime} \rightarrow t} T\left(t^{\prime}\right)(x+\xi y)=T(t)(x+\xi y)$ holds uniformly for $\xi \in C$, it follows that $\lim _{t^{\prime} \rightarrow t} d T\left(t^{\prime}\right)(x) y=d T(t)(x) y$.

Q.E.D.

Proof of Theorem 7.1. Fix an $m$ and let $t \geqq 0$ and $x \in D$. Since $T_{m}(t)$ $\in F(D)$ by Lemma 7.2 (a), we have that

$$
\begin{aligned}
& \quad h^{-1}\left\{T_{m}(t+h) x-T_{m}(t) x\right\}=h^{-1}\left\{T_{m}(t)\left[x+\left(T_{m}(h) x-x\right)\right]-T_{m}(t) x\right\} \\
& \quad=d T_{m}(t)(x)\left[h^{-1}\left(T_{m}(h) x-x\right)\right]+h^{-1} o\left(\left\|T_{m}(h) x-x\right\|\right) \quad \text { as } h \rightarrow+0 .
\end{aligned}
$$

Since $D \subset D\left(U_{m}\right)$, passing to the limit as $h \rightarrow+0,(d / d t) T_{m}(t) x=U_{m} T_{m}(t) x$ $=d T_{m}(t)(x) U_{m} x$. The derivative $(d / d t) T_{m}(t) x$ is strongly continuous in $t$, and so, we have that

$$
T_{m}(t) x-x=\int_{0}^{t} d T_{m}(s)(x) U_{m} x d s, \quad \text { for } t \geqq 0 \text { and } x \in D .
$$

On the other hand, $\left\|T_{m}(t)(x+y)-T_{m}(t) x-d T_{m}(t)(x) y\right\|=o(\|y\|)$ as $\|y\| \rightarrow 0$, and hence $\left\|d T_{m}(t)(x)(y /\|y\|)\right\| \leqq\left\|T_{m}(t)(x+y)-T_{m}(t) x\right\| /\|y\|+o(\|y\|) /\|y\| \leqq 1+o(\|y\|) /\|y\|$. This means that $\left\|d T_{m}(t)(x)\right\| \leqq 1$ for $t \geqq 0$ and $x \in D$. Thus, the set $\left\{d T_{m}(s)(x) U_{m} x ; s \geqq 0, m\right\}$ is bounded for $x \in D \cap D(A)$. Hence, Lemmas 7.1 (b) and 7.2 (a) imply that 


$$
\lim _{m \rightarrow \infty} d T_{m}(s)(x) U_{m} x=d T(s)(x) A x .
$$

By Lemma 7.2 (b), $d T(s)(x) A x$ is strongly continuous in $s \geqq 0$. Applying the dominated convergence theorem to (7.1), we have

$$
T(t) x-x=\int_{0}^{t} d T(s)(x) A x d s \quad \text { for } t \geqq 0 \text { and } x \in D \cap D(A) .
$$

Finally, we show that $d T(t)(x) A x=A T(t) x$ for $t \geqq 0$. By (7.2), $U_{m} T_{m}(t) x=$ $A J_{\eta_{m}} T_{m}(t) x \rightarrow d T(t)(x) A x$ strongly for each $t \geqq 0$. Since $\lim _{m \rightarrow \infty} J_{\eta_{m}} T_{m}(t) x=T(t) x$ and $A$ is closed, it follows that $T(t) x \in D(A)$ and $d T(t)(x) A x=A T(t) x$. Q.E.D.

\section{Waseda University}

\section{References}

[1] H. Brezis and A. Pazy, Semi-groups of nonlinear contractions on convex sets, to appear in J. Func. Anal.

[2] M. G. Crandall and A. Pazy, Semi-groups of nonlinear contractions and dissipative sets, J. Func. Anal., 3 (1969), 376-418.

[3] M. G. Crandall and A. Pazy, On accretive sets in Banach spaces, J. Func. Anal., 5 (1970), 204-217.

[4] J. R. Dorroh, A nonlinear Hille-Yosida-Phillips theorem, J. Func. Anal., 3 (1969), 345-353.

[5] J. R. Dorroh, Some classes of semi-groups of nonlinear transformations and their generators, J. Math. Soc. Japan, 20 (1968), 437-455.

[6] J.R. Dorroh, Semi-groups of nonlinear transformations, Michigan Math. J., 12 (1965), 317-320.

[7] E. Hille and R. Phillips, Functional Analysis and Semi-Groups, Amer. Math. Soc. Colloq. Publ., 31, 1957.

[8] Y. Kōmura, Nonlinear semigroups in Hilbert space, J. Math. Soc. Japan, 19 (1967), 493-507.

[9] Y. Kōmura, Differentiability of nonlinear semigroups, J. Math. Soc. Japan, 21 (1969), 375-402.

[10] T. Kato, Nonlinear semigroups and evolution equations, J. Math. Soc. Japan, 19 (1967), 503-520.

[11] T. Kato, Accretive operators and nonlinear evolution equations in Banach spaces, Proc. Symposium Nonlinear Func. Anal. Amer. Math. Soc. (1968) (to appear).

[12] I. Miyadera, On the convergence of nonlinear semigroups, Tôhoku Math. J., 21 (2) (1969), 221-236.

[13] I. Miyadera, On the convergence of nonlinear semigroups, II, J. Math. Soc. Japan, 21 (1969), 403-412.

[14] I. Miyadera and S. Oharu, Approximation of semi-groups of nonlinear operators, Tôhoku Math. J., 22 (1970), 24-47.

[15] S. Oharu, Note on the representation of semi-groups of nonlinear operators, Proc. Japan Acad., 42 (1966), 1149-1154.

[16] S. Oharu, Nonlinear semigroups in Banach spaces, to appear. 
[17] S. Oharu, A note on the generation of nonlinear semigroups in a locally convex space, Proc. Japan Acad., 43 (9) (1967), 847-851.

[18] W. V. Petryshyn, Remarks on fixed point theorems and their extensions, Trans. Amer. Math. Soc., 12 (1) (1967), 43-53.

[19] J.T. Schwartz, Nonlinear Functional Analysis, (1963-1964), Courant Inst. of Math. Sci., New York Univ.

[20] J. Watanabe, Semi-groups of nonlinear operators on closed convex sets, Proc. Japan Acad., 45 (4) (1969), 219-223. 